\title{
Central Tano Language
}

National Cancer Institute

\section{Source}

National Cancer Institute. Central Tano Language. NCI Thesaurus. Code C160958.

Languages of the Niger-Cong o family spoken in Ghana and Ivory Coast by the Akan people. 